\title{
4 Welche QM-Systeme gibt es in der ambulanten medizinischen und psychotherapeutischen Versorgung?
}

Julia Bellabarba

Das kontinuierliche Bestreben, Prozesse in einer Organisation zu verbessern, ist in erster Linie Ausdruck einer Haltung, die Wertschätzung und Respekt (gegenüber allen Mitarbeitern der Organisation selbst und gegenüber deren Kunden) ausdrückt. Die QM-Richtlinie des GBA stellt den Praxen frei, ob sie ein vorliegendes QM-System benutzen wollen oder die Umsetzung der Richtlinie anhand eines eigenständigen QM-Systems durchführen wollen, gewissermaßen mit einem „selbstgebastelten System“.

Wozu gibt es QM-Systeme? QM-Systeme strukturieren die QM-Arbeit und können sie dadurch erleichtern. Diese Systeme sind so aufgebaut, dass alle wesentlichen Ebenen der Qualität bearbeitet werden, und zwar unabhängig von der inhaltlichen Ausrichtung der Organisation.

Eine Übersicht der QM-Systeme, die sinnvollerweise in einer Praxis eingesetzt werden können, befindet sich in Anhang 3. Außerdem finden Sie eine detaillierte Gegenüberstellung der gängigen Praxis QM-Systeme unter: www. kbv.de/qep/11622.html.

Wenn eine Praxis, aus welchen Gründen auch immer, eine Zertifizierung anstrebt (wie in Kap. I.6 beschrieben), dann sollte sie sich für ein QM-System entscheiden, da eine Zertifizierung immer auf ein bestimmtes QM-System bezogen durchgeführt wird (Nota bene: man kann jedes, auch ein „selbstgebasteltes“ QM-System auf ein zertifizierungsfähiges QM-System umschreiben, hat dann aber mehr Aufwand). Von zentraler Bedeutung bei der Bewer- 
tung jedes QM-Systems für ambulante medizinische Einrichtungen sollte die Frage sein, ob das System vollständig mit der eingangs erwähnten GBA-Richtlinie übereinstimmt. Das bedeutet: sämtliche durch die GBA-Richtlinie formulierten Anforderungen müssen durch das QM-System abgedeckt sein. Tabelle 4 kann bei der Entscheidungsfindung hilfreich sein.

Tab. 4 Checkliste Bewertung der QM-Systeme

\begin{tabular}{l|l}
\hline Bewertung der QM-Systeme: Checkliste für Praxen & $\checkmark$ \\
\hline Bietet uns das QM-System die Möglichkeit der Zertifizierung? & \\
\hline Brauchen wir kooperationsbedingt dasselbe QM-System, das die Klinik hat, mit der wir \\
vertraglich verbunden sind? & \\
\hline Streben wir Kooperationsverträge an (integrierte Versorgung, MVZ, Netzwerkstruktur) und & \\
ergibt sich daraus die Notwendigkeit der Abstimmung mit den anderen Leistungserbringern? & \\
Ist es für uns günstig, dasselbe QM-System wie der Kooperationspartner zu haben? & \\
\hline Wollen wir ein QM-System, das für unsere Fachgruppe relevante Muster bereithält? & \\
Die Notwendigkeit von fachgruppenspezifischen Mustern wird unserer Einschätzung nach & \\
überbewertet, da Ärzte und Psychotherapeuten die Prozesshaftigkeit sämtlicher Leistungen \\
ihrer Praxis falsch einschätzen.
\end{tabular}

Alle in Anhang 3 aufgeführten QM-Systeme lassen sich auch auf (ärztliche und psychologische) psychotherapeutische Praxen anwenden. In Deutschland gibt es eine kleine Zahl von Psychotherapeuten, auch tiefenpsychologisch und psychoanalytisch ausgerichtet, die DIN EN ISO, QEP ${ }^{\circledR}$ und KTQ erfolgreich und mit Gewinn in ihren Einzelpraxen umgesetzt haben und deren Praxen auch zertifiziert wurden. Die Deutsche Psychotherapeutenvereinigung hat in Kooperation mit der KBV eine lizenzierte Version von $\mathrm{QEP}^{\circledR}$ für psychologische Psychotherapeuten entwickelt, ebenso die Vereinigung der Analytischen Kinder- und Jugendlichenpsychotherapeuten (leider vergriffen). Die übrigen $\mathrm{QM}$ Systeme für Psychotherapeuten bleiben im Umfang (Berücksichtigung sämtlicher psychotherapierelevanter Aspekte auf den drei Qualitätsebenen) und in der Zielsetzung (Richtlinienkonformität und Zertifizierungsfähigkeit) hinter den oben genannten Systemen zurück.

Die KBV und die Berufsgenossenschaft für Gesundheitsdienst und Wohlfahrtspflege haben in einem Kooperationsprojekt QEP-Qualitätsziele durch Arbeitsschutzziele ergänzt und eine Praxis ist bereits nach diesem erweiterten System zertifiziert worden. Sich der Thematik Arbeitsschutz und Arbeitssicherheit im Rahmen des praxisinternen Qualitätsmanagement besonders ausführlich zu widmen, macht vor allem für die medizinischen Organisatio- 
4 Welche QM-Systeme gibt es in der ambulanten medizinischen und psychotherapeutischen Versorgung?

nen Sinn, die viele Mitarbeiter und Auszubildende haben, ein hohes Potenzial an Gefährdung aufweisen und bisher wenig in diesem hochbrisanten Bereich geregelt haben.

In Anbetracht der Unübersichtlichkeit, die in den letzten Jahren auf dem Markt der QM-Systeme entstanden ist, wäre es wünschenswert, wenn die Qualität der Systeme selbst überprüft und zertifiziert würde, besonders in Hinblick auf die Vorgaben der Richtlinie. Ein systemübergreifendes Praxissiegel würde sowohl für die Kostenträger als auch für die Patienten Transparenz schaffen.

\section{Links}

www.q-m-a.de/6qmsysteme/3ktq/view

www.qualitaetspraxen.de

www.praxisassessment.de

www.q-m-a.de/6qmsysteme

www.q-m-a.de/7sonstigeinfos/checklisten/pdf/checkqma.pdf

www.kbv.de/qm

www.2din.de

www.efqm.org

www.deutsche-efam.org

www.ktq.de

www.europaeisches-praxisassessment.de

www.kbv.de/themen/qualitaetsmanagement.html

www.deutschepsychotherapeutenvereinigung.de 